\title{
Metástase hepática de adenocarcinoma colorretal com disseminação endobiliar: expressão das citoqueratinas 7,19 e 20
}

\author{
Hepatic metastasis of colorectal adenocarcinoma with endobiliary growth: \\ expression of cytokeratins 7, 19 and 20
}

Juliana Paschoal'; Adriana Caroli-Bottino²; Joaquim Ribeiro Filho33; Vera Lucia Pannain ${ }^{4}$

\begin{abstract}
unitermos
Neoplasias colorretais

Trato biliar

Metástase

Citoqueratina

Imuno-histoquímica

resumo

O crescimento endobiliar das neoplasias primárias e metastáticas do fígado é raro, com exceção dos colangiocarcinomas. Nas metástases esse comportamento pode suscitar dificuldade no diagnóstico diferencial com colangiocarcinomas quando são analisadas apenas as características morfológicas das neoplasias e/ou quando há associação com anormalidades que sugiram a origem biliar do tumor. Analisamos a imunoexpressão das citoqueratinas (CKs) 7, 19 e 20 em dois casos de metástase hepática de adenocarcinoma colorretal, com disseminação endobiliar, em fígados com adenoma e hamartomas biliares. Em ambos os casos, além da disseminação endobiliar da neoplasia, o epitélio biliar apresentava-se displásico. A neoplasia foi CK 20 positiva, CK 7 negativa, enquanto a CK 19 foi positiva no epitélio biliar normal, no displásico e na neoplasia. A CK 7 foi positiva no epitélio biliar, hamartomas e adenoma. Concluímos que o emprego da CK 7 e da CK 20 é importante em tumores com essa forma de disseminação, sobretudo quando associados aos hamartomas e adenomas biliares.
\end{abstract}

abstract

Endobiliary growth of primary and metastatic liver neoplasias is rare, with exception of cholangiocarcinoma. This behavior, when occurring in metastatic neoplasias, frequently challenges the differential diagnosis of cholangiocarcinomas, mainly when its analysis is based only in morphological characteristics of the neoplasias and/or there is association with abnormalities which suggest biliary origin of the tumor. We analyzed cytokeratins (CKs) 7, 19 and 20 imunoexpression in two cases of colorectal metastatic adenocarcinoma with endobiliary dissemination in livers with biliary adenoma and hamartoma. In both cases, in addition to endobiliary dissemination, there was also dysplasia in the biliary epithelium. The neoplasia was positive for $C K 20$, negative for $C K$ 7, while the normal epithelium, the dysplatic epithelium and the neoplasia were all positive for CK 19. The CK 7 was positive in the biliary epithelium, in hamartomas and adenoma. We concluded that the use of CK 7 and CK 20 is important in tumors with this type of dissemination, especially when they are associated with harmatomas and biliary adenomas. key words

Colorectal neoplasia

Biliary tract

Metastasis

Cytokeratins

Immunohistochemistry

1. Médica do Serviço de Anatomia Patológica do Hospital Universitário Clementino Fraga Filho da Universidade Federal do Rio de Janeiro (HUCFF/UFR))

2. Professora-adjunta do Departamento de Patologia da Faculdade de Medicina da UFR).

3. Professor-adjunto do Departamento de Cirurgia da Faculdade de Medicina da UFR).

4. Professora-adjunta do Departamento de Patologia da Faculdade de Medicina da UFR).

Trabalho realizado no Serviço da Anatomia Patológica/Departamento de Patologia da Faculdade de Medicina da UFR).

Apresentado no XIV Congresso Brasileiro de Patologia, em Florianópolis (SC), 2003. 


\section{Introdução}

O crescimento endobiliar das neoplasias primárias e metastáticas do fígado é raro, com exceção de alguns colangiocarcinomas $(\mathrm{CCs})^{(9,11)}$. Esse padrão de crescimento, quando ocorre em neoplasias metastáticas, pode levar a erro no diagnóstico, pois deverá ser diferenciado dos $\mathrm{CCs}^{(1,}$ $4,5,7,11,12)$. Os adenocarcinomas colorretais metastáticos (AMCs) para o fígado são os que mais freqüentemente apresentam esse comportamento ${ }^{(9,6)}$. Nesses casos, a diferenciação entre o CC e o AMC com disseminação endobiliar cria dificuldades para o patologista, quando a análise está baseada somente nas características morfológicas da neoplasia, sobretudo quando há associação com alterações no epitélio de ductos biliares, como displasias e/ou co-existência com anormalidades de vias biliares, por exemplo, o adenoma e o hamartoma, que sugerem a origem biliar do tumor ${ }^{(2,3,8)}$.

As citoqueratinas (CKs) são filamentos intermediários, relativamente específicos de um tipo de epitélio. O emprego de anticorpos específicos das diferentes isoformas de CKs pode ser útil para determinar a origem do órgão primário da neoplasia ${ }^{(13)}$. Avaliamos a expressão das CKs 7, 19 e 20 em dois casos de AMC, com disseminação endobiliar, associados a adenoma e hamartoma biliar.

\section{Relato dos casos}

Dois pacientes do sexo masculino, com história de colectomia por adenocarcinoma colorretal há dois anos, apresentaram nódulos hepáticos aos métodos de imagem, com hipótese diagnóstica de AMC, sendo então indicada a ressecção cirúrgica dos nódulos.

- Caso 1: segmento hepático medindo $8 \times 5 \times 4 \mathrm{~cm}$. Aos cortes, parênquima pardacento, elástico, compacto com área nodular, medindo $2,5 \times 2 \mathrm{~cm}$, firme-elástica.

- Caso 2: segmento hepático medindo $15 \times 9$ 9,5 × 5,5 $\mathrm{cm}$. Aos cortes, área brancacenta de contornos irregulares, medindo $3 \times 1,2 \mathrm{~cm}$, e ainda nódulo brancacento medindo $0,5 \times 0,3 \mathrm{~cm}$.

À microscopia, ambos exibiam neoplasia maligna epitelial composta por células colunares, com núcleos vesiculosos, pleomórficos e nucléolos evidentes, dispostas em arranjo glandular, compatível com adenocarcinoma metastático colorretal. A neoplasia, por vezes, exibia disseminação endobiliar, respeitando a membrana basal do ducto, porém com transição abrupta em relação ao seu epitélio próprio, que exibia displasia. Em ambos os casos foram observados espaços-porta apresentando proliferação de ductos biliares dilatados, irregulares e tortuosos, caracterizando hamartomas biliares e, no caso 2, se observou ainda adenoma biliar caracterizado pela proliferação de pequenos ductos biliares arredondados, revestidos por epitélio cubóide a cilíndrico simples, em meio a estroma fibroso denso.

Para o estudo imuno-histoquímico foi utilizada a técnica estreptoavidina-biotina peroxidase. Os cortes foram incubados com os seguintes anticorpos primários monoclonais de camundongo: CK 7 (isótipo lgG1, kappa, clone OV-TL 12/30), CK 19 (isótipo IgG1, kappa, clone BA17) e CK 20 (isótipo lgG 2a, kappa, clone Ks 20.8), todos Dako USA, e com a diluição de 1:50 em albumina tamponada, conforme otimizado no laboratório do Hospital Universitário Clementino Fraga Filho da Universidade Federal do Rio de Janeiro (HUCFF/UFRJ). Posteriormente, foi adicionado o anticorpo secundário LSAB System HRP (K0690) (Dako USA).

A reação foi revelada com o cromógeno $D A B$ e contracorado com hematoxilina. A imunorreatividade para esses anticorpos foi analisada nas células neoplásicas e no epitélio dos ductos biliares. Em ambos os casos, as células neoplásicas expressaram CKs 19 e 20 (Figuras 1 e 2) e foram negativas para CK 7 (Figura 3). As células do epitélio biliar, tanto normal como displásico, foram positivas para CKs 7 (Figura 3) e 19 (Figura 1) e negativas para CK 20 (Figura 2).

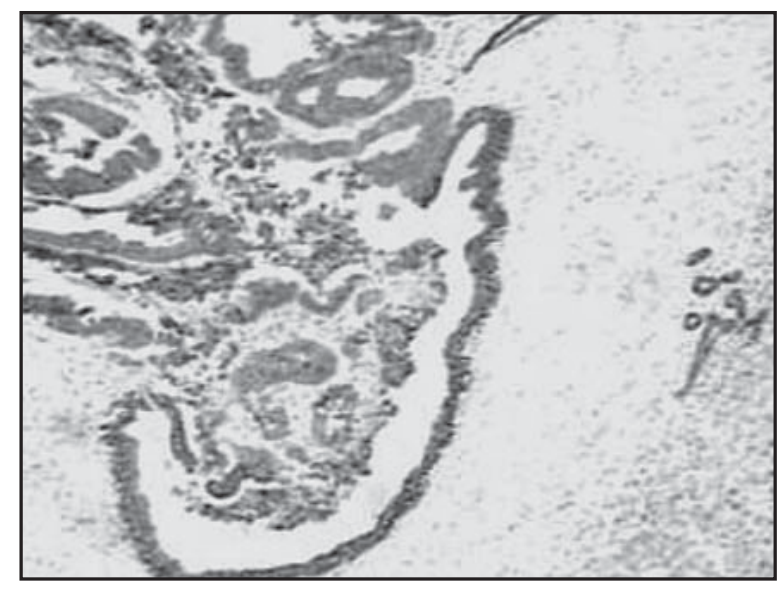

Figura 1 - Fígado com metástase de adenocarcinoma colorretal: positividade para CK 19 em células neoplásicas e em epitélio de ductos biliares 


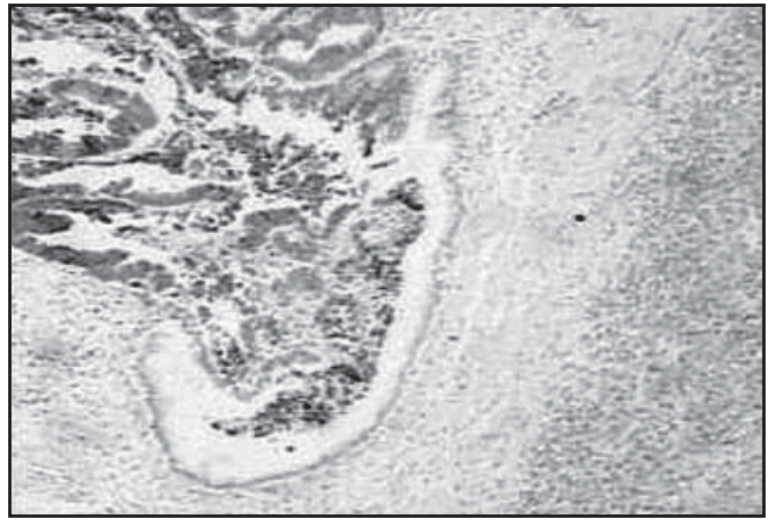

Figura 2 - Fígado com metástase de adenocarcinoma colorretal: positividade para anticorpo CK 20 apenas nas células neoplásicas

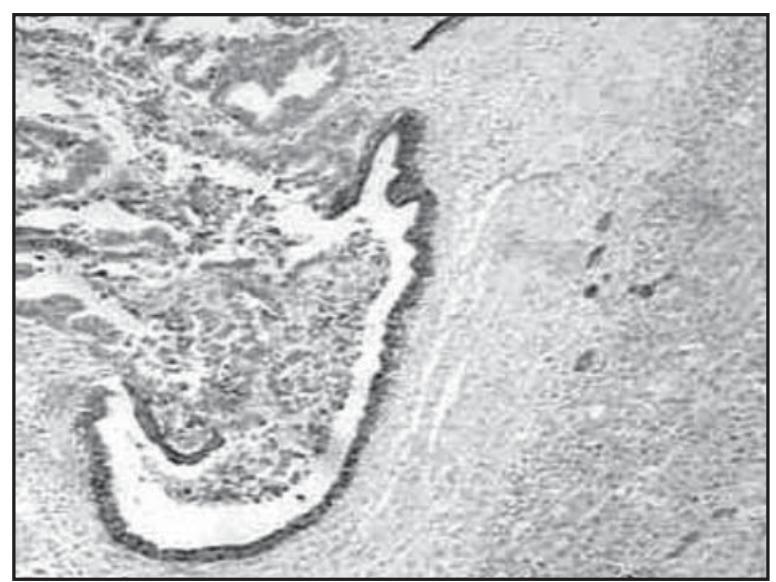

Figura 3 - Fígado com metástase de adenocarcinoma colorretal: positividade para CK 7 em células ductais

\section{Discussão}

As metástases hepáticas com crescimento endobiliar estão restritas a alguns relatos na literatura, sendo mais freqüentes as de origem colorretal ${ }^{(9,6)}$. A disseminação endobiliar do AMC pode ser de difícil diferenciação com o CC, quando são usados apenas métodos histológicos convencionais, tendo em vista que as características citológicas e arquiteturais dessas neoplasias por vezes se assemelham. Esse fato é agravado quando outras lesões das vias biliares, como o hamartoma e o adenoma, estão presentes, uma vez que essas podem estar associadas ao $\mathrm{CC}^{(7)}$. Vários autores destacam a importância de se utilizar o estudo imuno-histoquímico para a realização desse diagnóstico diferencial $(1,2,6,7,9,10,11,12)$.

Utilizam-se anticorpos específicos contra as CKs na caracterização dessas neoplasias, já que o fenótipo apresentado pelas células normais é geralmente mantido após a transformação neoplásica ${ }^{(6,2)}$. O epitélio dos ductos biliares expressa as CKs 7 e 19, enquanto as células do epitélio intestinal expressam a CK 20.

Maeda et al. ${ }^{(6)}$ demonstraram a importância do emprego simultâneo das CKs 7, 19 e 20 na diferenciação entre o CC e o AMC. Nos casos por eles estudados, a CK 7 foi positiva em $97 \%$ dos CCs, e a CK 20 negativa em 77\%, enquanto no AMC a CK 7 foi negativa em $93 \%$ e a 20 positiva em $90 \%$. Já a CK 19 foi difusamente positiva em $77 \%$ dos casos de CC, e em $64 \%$ dos casos de AMC.

Rullier et al. ${ }^{(9)}$ também enfatizaram a relevância do uso concomitante das CKs 7 e 20 nesse diagnóstico diferencial. O estudo mostrou uma variação na expressão da CK 20 nos CCs, que apresentou menor porcentagem de positividade nos tumores biliares periféricos, quando em comparação com os dos grandes ductos extra-hepáticos, respectivamente $47 \%$ e $86 \%$. Em todos os casos os AMCs foram CK 20 positivos, e somente $19 \%$ foram CK 7 positivos.

Shimonishi et $a l^{(10)}$, ressaltaram a relevância do uso de um painel imuno-histoquímico das $\mathrm{CKs}^{(7,8,18-20)}$ para o diagnóstico diferencial entre os CCs e vários tipos de adenocarcinomas metastáticos (AM), não apenas o colorretal. Os CCs foram negativos para CK 20 em 95\% dos casos e positivos para CK 7 em aproximadamente $97 \%$. Contudo, os autores observaram que houve particular variação da expressão da CK 20, pois a positividade para essa CK aumentava à medida que o tumor tornava-se menos diferenciado. Os AMCs tiveram 81\% de positividade para CK 20 e $86 \%$ de negatividade para CK 7. A positividade para CK 19 foi acima de $90 \%$ nos CCs, e variou de forma decrescente em relação aos tumores bem e pouco diferenciados. Os AMCs foram positivos para a CK 19 em 58\% dos casos, sendo a maior positividade observada nos de origem colorretal (71\%).

A exemplo dos autores citados, em nosso estudo as células neoplásicas que colonizavam ou não os ductos biliares foram CK 7 negativas/CK 20 positivas, caracterizando-as como de origem colorretal, e mostraram uma transição abrupta com o epitélio biliar normal ou displásico, cujo perfil imuno-histoquímico foi CK 7 positivo/CK 20 negativo, conforme também se observou nos hamartomas e no adenoma biliar.

Assim como Shimonishi et at. ${ }^{(10)}$ e Maeda et al. ${ }^{(6)}$ mostraram em seus estudos, nossos casos também demonstraram positividade para CK 19, tanto nas células do AMC quanto em células normais e displásicas ductais, mostrando que seu uso não auxiliou na diferenciação entre AMC e CC.

Concluímos que o emprego do estudo imuno-histoquímico com as CKs 7 e 20 é importante na diferenciação 
entre o AMC com disseminação endobiliar e o CC, sobretudo quando ocorre associação com outras lesões de vias biliares, como hamartomas e adenomas. Recomendamos ainda a pesquisa imuno-histoquímica das CKs 7 e 20, principalmente quando não se tem conhecimento do sítio primário da neoplasia.

\section{Referências}

I. BALATON,A.J. et al. Distinction between hepatocellular carcinoma, cholangiocarcinoma and metastatic carcinoma based on immunohistochemichal staining for carcinoembryonic antigen and for cytokeratin 19 on paraffin sections.J Pathol, v. 156, p. 305-10, 1988.

2. DANNA, E.J. et al. Diagnostic utility of the keratin profiles of hepatocellular carcinoma and cholangiocarcinoma. Am J Surg Pathol, v. 12, p. 187-97, 1988.

3. DEKKER, A. et al. Cholangiocarcinoma associated with multiple bileduct hamartomas of the liver. Dig Dis Sci, v. 34, p. 952-8, 1989.

4. FUCICHI, L.F. et al. Primary vs. metastatic hepatic carcinoma: an immunohistochemical study of 34 cases. Arch Pathol Lab Med, v. I 18, p. 927-9, 1994.

5. LAU, S.K. et al. Comparative immunohistochemical profile of hepatocelullar carcinoma, cholangiocarcinoma and metastatic adenocarcinoma. Hum Pathol, v. 33, n. 12, p. | |75-8|, 2002.

6. MAEDA, T. et al. The expression of cytokeratins 7, 19 and 20 in primary and metastatic carcinomas of the liver.Mod Pathol, v. 9, n. 9, p. 901-9, 1996.

7. RIOPEL, M.A. et al. Intrabiliary growth of metastatic colony adenocarcinoma: a pattern of intrahepatic spread easily confused with primary neoplasia of the biliary tract. Am J Surg Pathol, v. 21 , n. 9, p. 1030-6, 1997.

8. ROCKEN, C. et al. Cholangiocarcinoma occurring in a liver with multiple bile ducts hamartomas (von Meyenburg complexes). Arch Pathol Lab Med, v. 124, n. I, p. 1704-6, 2000.

9. RULLIER, A. et al. Cytokeratin 7 and 20 expression in cholangiocarcinomas varies along the biliary tract but still differs from that in colorectal carcinoma metastasis. Am J Surg Pathol, v. 24, n. 6, p. 870-6, 2000.

10. SHIMONISHI,T. et al. Cytokeratin profile relates to histological subtypes and intrahepatic location of intrahepatic cholangiocarcinoma and primary sites of metastatic adenocarcinoma of liver. Histopathology, v. 37, p 55-63, 2000.

I I.TAKAMATSU, S. et al. Liver metastasis from rectal cancer with proeminent intrabile duct growth. Pathol Int, v. 54, p. 440-5 2004.

12. UEHARA, K. et al. Intrabiliary polypoid growth of liver metastasis from liver colonic adenocarcinoma with minimal invasion of liver parenchyma. J Gastroenterol, v. 39, p. 72-5, 2004.

13. WENER, B.M.D. et al. Uso prático da imuno-histoquímica em patologia cirúrgica. J Bras Patol, v. 4I, n. 5, p. 353-64, 2005. 